whether patients reported to have fatty liver on USS, irrespective of clinical indication, were adequately assessed by testing of liver function tests (LFTs) and if abnormal, subsequently referred to a specialist clinic.

Methods A single centre, retrospective analysis of all patients who underwent USS over a 5-month period (January-May 2011) at Chase Farm Hospital was performed. Patients who had LFTs within 8 weeks of USS were said to have had their LFTs checked appropriately. Data were obtained from radiology reports via PACS/EPR reporting systems.

Results 258 patients were investigated over the audit period. 69 $(26.7 \%)$ patients (42 male, 27 female), median age 58 years (25-91 years) were reported to have fatty liver on ultrasound. 52 $(75.3 \%)$ of these patients had their LFTs checked of which 37 $(71.2 \%)$ were abnormal. $12(17.3 \%)$ patients with fatty liver on ultrasound were formally seen in a specialist clinic. Over half of patients $(27,51.9 \%)$ with fatty liver and abnormal LFTs were never seen in a specialist clinic.

Conclusion A quarter of patients with USS diagnosis of fatty liver did not have their LFTs checked potentially missing an opportunity to monitor for complications of NAFLD. While only the tip of the iceberg of NAFLD patients are referred to secondary care, a large portion of the iceberg goes unnoticed even on surfacing. An increased awareness of NAFLD needs to relayed to all healthcare professionals including radiologists and general practitioners coming into contact with this "iceberg."

Competing interests None declared.

\section{PWE-293 LOW GLYCAEMIC INDEX DIETARY INTERVENTION FOR PATIENTS WITH NON-ALCOHOLIC FATTY LIVER DISEASE IN THE GENERAL POPULATION-A RANDOMISED CONTROLLED TRIAL}

doi:10.1136/gutjnl-2012-302514d.293

${ }^{1}$ V W-S Wong, ${ }^{*}$ R S-M Chan, ${ }^{1} \mathrm{G}$ L-H Wong, ${ }^{2} \mathrm{~B}$ H-K Cheung, ${ }^{3}$ W C-W Chu, ${ }^{1} \mathrm{H}$ L-Y Chan, ${ }^{1} \mathrm{~J}$ Woo. ${ }^{1}$ Department of Medicine and Therapeutics, The Chinese University of Hong Kong, Hong Kong, China; ${ }^{2}$ Centre for Nutritional Studies, The Chinese University of Hong Kong, Hong Kong, China; ${ }^{3}$ Department of Imaging and Interventional Radiology, The Chinese University of Hong Kong, Hong Kong, China

Introduction Low glycaemic index diet improves insulin sensitivity and adiposity. Its effect on non-alcoholic fatty liver disease (NAFLD) is unclear.

Methods This was a single-blind, randomised controlled trial of NAFLD patients aged 18-70 years who were recruited through population screening. All patients were instructed to exercise around $90 \mathrm{~min} /$ week, and were randomised to participate in a low glycaemic index dietary intervention programme or receive usual care. The dietary intervention programme was led by dietitians and consisted of education on food components, interpretation of food labels, food exchanges, healthy eating out techniques and cooking methods. The primary endpoint was the proportion of patients with resolution of NAFLD at month 12, defined as intrahepatic triglyceride content (IHTG) $<5 \%$ by proton-magnetic resonance spectroscopy.

Results At the time of analysis, 104 patients (51 in the intervention group and 53 in the control group) had completed month 12 assessment. The adherence to the intervention programme was excellent, with attendance over $80 \%$. Resolution of NAFLD occurred in $35(69 \%)$ patients in the intervention group and $11(21 \%)$ in the control group $(p<0.001)$. IHTG decreased from $11.7 \pm 6.2 \%$ to $4.8 \pm 4.6 \%$ in the intervention group but remained static from $11.7 \pm 6.9 \%$ to $9.7 \pm 6.7 \%$ in the control group (mean difference in IHTG change $4.9 \%$; $95 \%$ CI $2.6 \%$ to $7.1 \%$ ). Reduced body mass index (BMI) was observed in the intervention group first at month 3 and maintained through month 12. At month 12, BMI decreased by
$9.2 \pm 7.8 \%$ from baseline in the intervention group, compared to $1.1 \pm 5.5 \%$ in the control group $(\mathrm{p}<0.001)$. By multivariate analysis, dietary intervention (OR 4.2; 95\% CI 1.1 to 15.4), baseline IHTG (OR $0.82 ; 95 \%$ CI 0.72 to 0.93 ) and percentage change in BMI (OR $0.78 ; 95 \%$ CI 0.68 to 0.89 ) were independent factors associated with NAFLD resolution.

Conclusion Low glycaemic index dietary intervention is effective in reducing liver fat in NAFLD patients in the general population. (This study was supported in part by the Nutritional Research Foundation of the UK and the Centre for Nutritional Studies, Faculty of Medicine, The Chinese University of Hong Kong.

Clinical trial registration number ClinicalTrials.gov number, NCT00868933.

Competing interests None declared.

\section{PWE-294 MICROPARTICLE DEPENDENT PROCOAGULANT ACTIVITY AND THROMBIN GENERATION IS INCREASED IN PATIENTS WITH CIRRHOSIS INDUCED COAGULOPATHY}

doi:10.1136/gutjnl-2012-302514d.294

${ }^{1} \mathrm{~V}$ Jairath, ${ }^{2} \mathrm{P}$ Harrison, ${ }^{3} \mathrm{~S}$ Stanworth, ${ }^{1} \mathrm{~J}$ Collier, ${ }^{3} \mathrm{M}$ Murphy, ${ }^{1} \mathrm{E}$ Barnes. ${ }^{1} \mathrm{~J} o h n$ Radcliffe Hospital, Oxford, UK; ${ }^{2}$ Oxford Haemophilia and Thrombosis centre, Oxford, UK; ${ }^{3} \mathrm{NHS}$ Blood and Transplant, Oxford, UK

Introduction Recent data suggests stable cirrhotics may have a hypercoagulable phenotype. Microparticles (MPs) are submicron plasma particles formed by the exocytic budding of cell membranes and play an important role in haemostasis due to phosphatidylserine (PS) surface expression which provides a phospholipid surface for assembly of coagulation enzymes and/or the expression of tissue factor (TF), the primary initiator of coagulation. To determine whether MPs may contribute to this hypercoagulable phenotype, we assessed microparticle associated functional procoagulant and phenotypic characteristics in cirrhotics.

Methods 72 consecutive cirrhotics and 30 healthy volunteers were recruited. Platelet free plasma (PFP) was prepared by two centrifugations and MP-free plasma (MP-FP) by filtration of PFP through a $200 \mathrm{~nm}$ microparticle filtration unit. Microparticle (MP) associated procoagulant activity (PCA) was measured using the STA Procoag PPL (phospholipid) assay (Stago Diagnostics) and MP associated thrombin generation (TG) measured using the calibrated automated thrombogram (CAT). For the CAT assay TG was initiated by adding $\mathrm{CaCl}_{2}$ and $1 \mathrm{pM}$ tissue factor, but no phospholipid (PRP reagent), therefore TG was dependent upon phospholipid present in the sample. Flow cytometry (LSRII) was used to determine MP size, number and cellular origin using marker specific antibodies.

Results PFP from cirrhotics generated significantly more thrombin than healthy volunteers reflected in the ETP (1374.3 vs $1142.6 \mathrm{nM}$ / min, $\mathrm{p}=0.04)$, the peak (101.0 vs $66.5 \mathrm{nM}, \mathrm{p}=0.001)$ and a shorter time to peak ( 13.0 vs $14.2 \mathrm{~min}, \mathrm{p}=0.03$ ). Similarly, MP associated PCA was significantly increased in cirrhotics (65.9 $\pm 13.2 \mathrm{~s})$, compared to healthy volunteers $(74.6 \pm 13.9 \mathrm{~s}, \mathrm{p}=0.005)$. Following filtration of MPs $>200 \mathrm{nM}$ in size, there was a large reduction in ETP and peak in both cirrhotics and healthy volunteers, with prolongation of both the time to peak and PPL time. There was significant inverse correlation between the PPL assay and parameters of the TG test [ETP $(r=-0.57, p<0.001)$, Peak $(r=-0.43, p<0.001)$ ]. Cirrhotic patients had high levels of Annexin V binding PS positive MPs compared to controls (1412 vs 279 per $\mathrm{u} / \mathrm{l}, \mathrm{p}<0.05$ ).

Conclusion Microparticle dependent procoagulant activity and thrombin generating capacity is increased in plasma from cirrhotics. High levels of annexin-V positive procoagulant MPs are a likely key and previously undescribed mechanism contributing to the hypercoaguable phenotype observed in cirrhotics.

Competing interests None declared. 